\title{
Opioid-Induced Long-Term Potentiation in the Spinal Cord Is a Presynaptic Event
}

\author{
Hong-Yi Zhou, ${ }^{1 *}$ Shao-Rui Chen, ${ }^{1 *}$ Hong Chen, ${ }^{1}$ and Hui-Lin Pan ${ }^{1,2}$ \\ ${ }^{1}$ Department of Anesthesiology and Perioperative Medicine, The University of Texas M. D. Anderson Cancer Center, Houston, Texas 77030, and ${ }^{2}$ Program \\ in Neuroscience, The University of Texas Graduate School of Biomedical Sciences, Houston, Texas 77225
}

Opioids remain the mainstay of treatment for severe pain, but the associated hyperalgesia and tolerance are significant impediments to achieving adequate pain relief with opioids. Here we show that in the spinal cord, brief application of the $\mu$-opioid receptor agonist $\left(\mathrm{D}-\mathrm{Ala}^{2}, \mathrm{~N}-\mathrm{Me}-\mathrm{Phe}^{4}, \mathrm{Gly}^{-\mathrm{ol}^{5}}{ }^{5}\right.$-enkephalin $(\mathrm{DAMGO})$ at $1 \mu \mathrm{M}$, but not at $1-10 \mathrm{~nm}$, caused an initial decrease followed by a large and long-lasting increase in the amplitude of monosynaptic EPSCs evoked from the dorsal root in 50\% of lamina I and II neurons. However, postsynaptic dialysis of the G-protein inhibitor had no effect on DAMGO-induced initial inhibition and long-term potentiation (LTP) in either lamina I or II neurons. DAMGO-induced LTP was associated with an increase in the paired-pulse depression ratio. Furthermore, DAMGO application and washout induced an initial decrease followed by a persistent increase in the frequency of miniature EPSCs. Bath application, but not postsynaptic dialysis, of an NMDA receptor antagonist or a calcium chelator abolished DAMGO-induced LTP. Strikingly, ablation of TRPV1-expressing primary afferents not only eliminated DAMGO-induced LTP but also prolonged DAMGOinduced inhibition of the miniature and evoked EPSCs (i.e., long-term depression). Thus, our study strongly suggests that TRPV1expressing primary afferents play a prominent role in opioid-induced presynaptic LTP, which challenges a previous report suggesting the postsynaptic nature of this opioid-induced LTP. This excitatory effect of opioids on primary afferents can counteract the inhibitory effect of opioids on synaptic transmission at the spinal level and is likely involved in opioid-induced hyperalgesia and tolerance.

\section{Introduction}

The $\mu$-opioid receptor agonists remain the most effective analgesics for the treatment of severe pain. However, opioids can paradoxically elicit abnormal pain or hyperalgesia. Opioid-induced hyperalgesia has been demonstrated in animal models and in patients (Devulder, 1997; Célèrier et al., 2000). Even brief exposure to fentanyl or morphine can induce long-lasting hyperalgesia (Célèrier et al., 2000; Van Elstraete et al., 2005). This abnormal opioid-induced pain differs from the original pain complaint in presentation, location, and quality (Devulder, 1997). The increased pain sensitivity requires the use of additional opioids to sustain the analgesic effects and, consequently, can be interpreted as analgesic tolerance. These two important phenomena may occur through similar mechanisms. For example, animals receiving repeated morphine injections show a progressive reduction in the nociceptive threshold, which occurs in parallel with the development of tolerance (Mao et al., 1994; Chen et al., 2007). Also, treatments that reduce opioid-induced hyperalgesia, such as TRPV1 blockers (Chen et al., 2008) and NMDA receptor antag-

\footnotetext{
Received Nov. 25, 2009; revised Feb. 5, 2010; accepted Feb. 16, 2010.

This work was supported by the National Institutes of Health (Grants GM64830 and NS45602) and by the N. G. and Helen Hawkins endowment (to H.L.P.). We thank Dr. Xiudong Lei in the Department of Biostatistics for statistical consultations.

*H.-Y.Z. and S.-R.C. contributed equally to this work.

Correspondence should be addressed to Hui-Lin Pan, Department of Anesthesiology and Perioperative Medicine, The University of Texas M. D. Anderson Cancer Center, 1515 Holcombe Boulevard, Unit 110, Houston, TX 77030 4009. E-mail: huilinpan@mdanderson.org.

DOI:10.1523/JNEUROSCI.5857-09.2010

Copyright $\odot 2010$ the authors $\quad 0270-6474 / 10 / 304460-07 \$ 15.00 / 0$
}

onists (Trujillo and Akil, 1991; Mao et al., 1994), attenuate opioid tolerance. However, the unifying cellular and molecular mechanisms of opioid-induced hyperalgesia and tolerance remain unclear.

The $\mu$-opioid receptors are distributed in the dorsal root ganglion neurons, the spinal cord dorsal horn, and discrete regions of the brain. At the spinal cord level, $\mu$-opioid receptor agonists likely produce analgesia by presynaptic attenuation of the primary afferent input and by postsynaptic inhibition of dorsal horn neurons (Kohno et al., 1999; Marker et al., 2005; Zhou et al., 2008a). However, the neuronal circuitry and endogenous mechanisms relevant to opioid-induced hyperalgesia and tolerance remain poorly understood. The key unanswered questions are where and how opioids initiate plasticity in the nervous system, which leads to opioid-induced hyperalgesia and tolerance. This problem has not been adequately studied in the nociceptive circuitry where native opioid receptors are expressed and, thus, the specific cellular sites and signaling mechanisms involved in the initiation of opioid-induced hyperalgesia and tolerance are not fully known.

During the course of our recent study, we observed a rebound increase in glutamatergic input to some lamina II neurons during the washout period of the $\mu$-opioid receptor agonist (Zhou et al., 2008a). In this study, we determined the cellular origin of increased glutamatergic synaptic transmission after acute opioid exposure using a spinal cord slice preparation that possessed native $\mu$-opioid receptors and preserved the intrinsic connection between primary afferents and dorsal horn neurons. Contrary to a previous report showing a postsynaptic origin of opioid 
withdrawal-induced long-term potentiation (LTP) (Drdla et al., 2009), we provide new evidence showing that this LTP can originate presynaptically in the spinal cord, especially from TRPV1expressing primary afferents. Correctly defining the cellular origin of opioid-induced LTP is critical because it impacts whether we should focus on primary sensory neurons or spinal second-order neurons in our future research to uncover the mechanisms of opioid-induced hyperalgesia and tolerance.

\section{Materials and Methods}

Animals and resiniferatoxin treatment. Male Sprague Dawley rats (7-8 weeks old; Harlan) were used in our study. All experiments were approved by the Animal Care and Use Committee at the University of Texas M. D. Anderson Cancer Center and conformed to the guidelines of $\mathrm{Na}$ tional Institutes of Health's Guide for the Care and Use of Laboratory Animals. Rats received a single intraperitoneal injection of resiniferatoxin (RTX; $200 \mu \mathrm{g} / \mathrm{kg}$; LC Laboratories) dissolved in a mixture of $10 \%$ Tween 80 and 10\% ethanol in normal saline while under anesthesia with 2-3\% isoflurane (Chen and Pan, 2006; Zhou et al., 2009). Rats in the control group received vehicle injection alone. Ablation of TRPV1expressing primary afferents in RTX-treated rats was confirmed by diminished paw withdrawal responses to a noxious heat stimulus and by depletion of TRPV1-immunoreactive neurons in the dorsal root ganglion using immunocytochemical labeling as described previously (Chen and Pan, 2006; Zhou et al., 2009). The final electrophysiological experiments were conducted 7-10 d after the RTX or vehicle injections.

Spinal cord slice preparation and electrophysiological recordings. Under $2-3 \%$ isoflurane anesthesia, the lumbar segment of the rat spinal cord was removed by laminectomy. The spinal cord tissue was immediately placed in ice-cold sucrose artificial CSF (aCSF) presaturated with $95 \% \mathrm{O}_{2}$ and $5 \% \mathrm{CO}_{2}$. The sucrose aCSF contained the following (in $\mathrm{mm}$ ): 234 sucrose, $3.6 \mathrm{KCl}, 1.2 \mathrm{MgCl}_{2}, 2.5 \mathrm{CaCl}_{2}, 1.2 \mathrm{NaH}_{2} \mathrm{PO}_{4}, 12.0$ glucose, and $25.0 \mathrm{NaHCO}_{3}$. Transverse spinal cord slices $(400 \mu \mathrm{m})$ were cut in the ice-cold sucrose aCSF using a vibratome and preincubated in Krebs solution oxygenated with $95 \% \mathrm{O}_{2}$ and $5 \% \mathrm{CO}_{2}$ at $34^{\circ} \mathrm{C}$ for at least $1 \mathrm{~h}$ before recording. The Krebs solution contained the following (in $\mathrm{mM}$ ): 117.0 $\mathrm{NaCl}, 3.6 \mathrm{KCl}, 1.2 \mathrm{MgCl}_{2}, 2.5 \mathrm{CaCl}_{2}, 1.2 \mathrm{NaH}_{2} \mathrm{PO}_{4}, 11.0$ glucose, and 25.0 $\mathrm{NaHCO}_{3}$.

Recordings of EPSCs were performed using the whole-cell voltageclamp method as we described previously (Li et al., 2002; Zhou et al., 2008a). The spinal cord slice was continuously perfused with Krebs solution at $5.0 \mathrm{ml} / \mathrm{min}$ at $34^{\circ} \mathrm{C}$ maintained by an inline solution heater. The lamina II was identified as a distinct translucent band across the superficial dorsal horn under a microscope with transmitted illumination. Neurons in the outer zone of lamina II were studied in most of the experiments because they receive input from both TRPV1- and nonTRPV1-expressing C-fiber afferent terminals (Pan et al., 2003; Pan and Pan, 2004; Chen and Pan, 2006). In some experiments, large-diameter lamina I neurons were also selected for recording. Lamina I and II neurons in the slice were identified on a fixed-stage microscope using differential interference contrast/infrared illumination. EPSCs were recorded with an electrode (impedance was 5-8 $\mathrm{M} \Omega$ ) filled with the following internal solution (in $\mathrm{mM}$ ): 135.0 potassium gluconate, $5 \mathrm{KCl}, 2.0 \mathrm{MgCl}_{2}$, $0.5 \mathrm{CaCl}_{2}, 5.0$ HEPES, 5.0 EGTA, 5.0 ATP-Mg, and 0.5 Na-GTP, adjusted to $\mathrm{pH} 7.2-7.3$ with $1 \mathrm{M} \mathrm{KOH} \mathrm{(290-300} \mathrm{mOsm).} \mathrm{EPSCs} \mathrm{were} \mathrm{evoked} \mathrm{by}$ electrical stimulation $(0.6 \mathrm{~mA}, 0.2 \mathrm{~ms})$ of the attached dorsal root, and monosynaptic EPSCs were identified on the basis of the constant latency and the absence of conduction failure of evoked EPSCs in response to a $20 \mathrm{~Hz}$ electrical stimulation as we described previously (Li et al., 2002; Zhou et al., 2008a).

To determine the paired-pulse depression or facilitation, two EPSCs were evoked by a pair of stimuli given at $50 \mathrm{~ms}$ intervals. The paired-pulse ratio was expressed as the ratio of the amplitude of the second synaptic response to the amplitude of the first synaptic response. The AMPA receptor-mediated EPSCs were recorded at a holding potential of -60 $\mathrm{mV}$ in the presence of $10 \mu \mathrm{M}$ bicuculline and $2 \mu \mathrm{M}$ strychnine. The NMDA receptor-mediated EPSCs were recorded at $+40 \mathrm{mV}$ in the presence of $10 \mu \mathrm{M}$ 6-cyano-7-nitroquinoxaline-2,3-dione (CNQX), $10 \mu \mathrm{M}$ bicuculline, and $2 \mu \mathrm{M}$ strychnine (Zhou et al., 2008b). To record the miniature EPSCs (mEPSCs), $0.5 \mu \mathrm{M}$ tetrodotoxin was added to the perfusion solution. In some experiments, to examine the presynaptic effects of the $\mu$-opioid receptor agonist (D-Ala ${ }^{2}, \mathrm{~N}-\mathrm{Me}-\mathrm{Phe}^{4}, \mathrm{Gly}_{-} \mathrm{ol}^{5}$ )enkephalin (DAMGO), we used the following internal solution (in $\mathrm{mM}$ ): $110 \mathrm{Cs}_{2} \mathrm{SO}_{4}$, 5 tetraethylammonium, $2.0 \mathrm{MgCl}_{2}, 0.5 \mathrm{CaCl}_{2}, 5.0$ HEPES, 5.0 EGTA, 5.0 ATP-Mg, 0.5 Na-GTP, 1 guanosine 5'-O-(2thiodiphosphate) (GDP- $\beta$-S), and 10 lidocaine $N$-ethyl bromide that had been adjusted to $\mathrm{pH} 7.2-7.3$ with $1 \mathrm{M} \mathrm{CsOH} \mathrm{(290-300} \mathrm{m} \Omega \mathrm{sm})$. In this case, GDP- $\beta-S$ and $\mathrm{Cs}^{2+}$ were used to inhibit the postsynaptic effect of the opioid receptor agonist (Zhou et al., 2008a).

The postsynaptic G-protein-coupled inwardly rectifying $\mathrm{K}^{+}$channel (GIRK) currents were recorded from lamina II neurons at a holding potential of $-60 \mathrm{mV}$ using the pipette internal solution containing the following (in $\mathrm{mm}$ ): 135.0 potassium gluconate, $5.0 \mathrm{KCl}, 2.0 \mathrm{MgCl}_{2}, 0.5$ $\mathrm{CaCl}_{2}$, 5.0 HEPES, 5.0 EGTA, 5.0 ATP-Mg, 0.5 Na-GTP; the solution was adjusted to pH 7.2-7.4 with $1 \mathrm{~m} \mathrm{KOH}(290-300 \mathrm{~m} \Omega s m)$ (Zhou et al., 2008a). Signals were recorded using an amplifier (MultiClamp700B; Molecular Devices), filtered at $1-2 \mathrm{kHz}$, digitized at $10 \mathrm{kHz}$, and stored into a computer. DAMGO, D-Phe-Cys-Trp-Arg-Thr-Pen-Thr-NH2 (CTAP), BAPTA, GDP- $\beta$-S, CNQX, 2-amino-5-phosphonopentanoic acid (AP$5)$, and MK-801 were obtained from Sigma-Aldrich. Bicuculline, strychnine, and BAPTA-AM were purchased from Tocris Bioscience Cookson.

Data analysis. Data are presented as mean \pm SEM. The amplitudes of evoked EPSCs and GIRK currents were analyzed offline with Clampfit 9.2. The mean amplitude of 10 consecutive evoked EPSCs before opioid application was used as the control, and the amplitudes of evoked EPSCs during DAMGO application and the washout period were normalized to the control value for each cell. The frequency and amplitude of mEPSCs were analyzed offline using a peak detection program (MiniAnalysis; Synaptosoft) and averaged every $3 \mathrm{~min}$ during the control, DAMGO application, and the washout periods. The raw data were used to test the drug effects on the evoked EPSCs and mEPSCs for statistical differences using repeated-measures ANOVA followed by Dunnett's post hoc test or nonparametric ANOVA (Kruskal-Wallis test). $p<0.05$ was considered statistically significant.

\section{Results}

Presynaptic $\mu$-opioid receptors contribute to opioid-induced inhibition of glutamatergic transmission and LTP in the spinal cord

In 10 of 19 (53\%) lamina II neurons examined, bath application of the $\mu$-opioid receptor agonist DAMGO ( $1 \mu \mathrm{M})$ for $3 \mathrm{~min}$ initially reduced the amplitude of monosynaptic EPSCs evoked from the dorsal root. Surprisingly, after washout of DAMGO, there was a significant and persistent increase in the amplitude of monosynaptic EPSCs in these neurons (Fig. 1A). Also, the opioid-induced inhibition and potentiation of monosynaptic EPSCs were associated with an increase in the paired-pulse depression ratio (Fig. $1 \mathrm{~B}$ ). In the other nine lamina II neurons, DAMGO acutely inhibited only the evoked monosynaptic EPSCs. At the end of the recording period, bath application of 10 $\mu \mathrm{M}$ CNQX completely blocked evoked EPSCs. The specific $\mu$-opioid receptor antagonist CTAP (1 $\mu \mathrm{M})$ abolished the DAMGO-induced acute inhibition and LTP (Fig. 1C).

It has been reported that low concentrations (1-10 nM) of opioids increase, whereas high concentrations $(1 \mu \mathrm{M})$ of opioids decrease, the action potential duration in primary afferent neurons (Shen and Crain, 1989). Thus, we determined whether the LTP during DAMGO washout was a result of increased excitability of primary afferent neurons caused by low concentrations of opioids. Bath application of 1-10 nM DAMGO did not significantly alter the amplitude of monosynaptic EPSCs during DAMGO application or washout in any of lamina II neurons tested (Fig. 1D). 
To determine whether postsynaptic $\mu$-opioid receptors were involved in this opioid-induced LTP, we included a general G-protein inhibitor, GDP- $\beta$-S, in the pipette internal solution. In 12 lamina II neurons recorded without GDP- $\beta$-S, bath application of $1 \mu \mathrm{M}$ DAMGO produced an outward current with amplitude of $35.2 \pm 2.8 \mathrm{pA}$. Intracellular dialysis of 1 mM GDP- $\beta$-S abolished the postsynaptic GIRK currents elicited by DAMGO in all 13 lamina II neurons (Fig. 2). However, the presence of intracellular GDP- $\beta$-S failed to significantly reduce the magnitude and incidence of the DAMGOinduced LTP and had no significant effect on the acute inhibition by DAMGO in lamina II neurons (Fig. 2).

We also determined whether presynaptic $\mu$-opioid receptors contribute to LTP induced after cessation of DAMGO application in lamina I neurons. In 11 of $21(52 \%)$ neurons tested, bath application of $1 \mu \mathrm{M}$ DAMGO for 3 min acutely inhibited evoked monosynaptic EPSCs and induced LTP after cessation of DAMGO application (Fig. 3A). Postsynaptic dialysis of $1 \mathrm{~mm}$ GDP- $\beta$-S failed to alter the inhibition or the magnitude and incidence of the DAMGO-induced LTP in lamina I neurons (Fig. 3B). These results strongly suggest that $\mu$-opioid receptors expressed on primary afferents mediate opioid-induced acute depression and LTP in both lamina I and II neurons.

\section{TRPV1-expressing primary afferents} are critical for opioid-induced LTP in the spinal cord

We next determined the contribution of TRPV1-expressing primary afferents to opioid-induced LTP in the spinal cord. Systemic RTX treatment selectively eliminates TRPV1-expressing primary afferents in adult rats but does not affect the intrinsic TRPV1-expressing neurons in the spinal cord and postsynaptic $\mu$-opioid receptor function (Chen and Pan, 2006; Zhou et al., 2008a, 2009). TRPV1-expressing neurons were eliminated in dorsal root ganglia removed from RTX-treated rats (Fig. 4A). Strikingly, ablation of the TRPV1-expressing primary afferents not only abolished the persistent increases in the amplitude of evoked EPSCs after DAMGO washout but also prolonged DAMGO-induced inhibition of the evoked monosynaptic EPSCs in lamina II neurons (Fig. 4B).

Furthermore, brief application of DAMGO $(1 \mu \mathrm{M})$ initially reduced the frequency of mEPSCs but induced an increase in the mEPSC frequency during the washout period in $50 \%$ of the lamina II neurons tested (Fig. 5A). DAMGO application had no significant effects on the amplitude of mEPSCs (control, $20.73 \pm 1.49$ pA; DAMGO application, $20.05 \pm 1.25 \mathrm{pA}$; and $20 \mathrm{~min}$ after DAMGO washout, $20.61 \pm 1.26 \mathrm{pA} ; p>0.05)$. Additionally, removing TRPV1-expressing primary afferents not only abolished the persistent increases in the frequency of mEPSCs after DAMGO washout but also caused a prolonged inhibition of the mEPSC frequency by DAMGO in all lamina II neurons tested
(Fig. 5B). These results further suggest that both the acute depression and LTP produced by $\mu$-opioid receptor activation are presynaptic events.

\section{Presynaptic NMDA receptors and increased $\mathrm{Ca}^{2+}$ contribute} to opioid-induced LTP in the spinal cord

Opioid-induced hyperalgesia and tolerance can be inhibited by NMDA receptor antagonists (Mao et al., 1994; Célèrier et al., 2000). However, it is not clear whether presynaptic or postsynaptic (e.g., primary afferents vs dorsal horn neurons) NMDA receptors are involved. Postsynaptic intracellular application of the NMDA channel blocker MK-801 (1 mM) in lamina II neurons for $15 \mathrm{~min}$ before bath application of $1 \mu \mathrm{M}$ DAMGO had no significant effect on the magnitude and incidence of the DAMGO-induced LTP (Fig. 6A). Intracellular application of 1 mM MK-801 for 10-15 min completely blocked the NMDA currents evoked from the dorsal root. However, intracellular dialysis of $10 \mu \mathrm{M}$ MK-801 had no effect on NMDA currents in all of the lamina II neurons examined (Fig. 6B). Unlike MK-801, which noncompetitively blocks the NMDA channel pore, AP-5 is a competitive NMDA receptor antagonist that blocks the glutamate binding site of the NMDA receptors. Pretreatment with bath application of $50 \mu \mathrm{M}$ AP- 5 prevented LTP induction by DAMGO (Fig. 6C). Also, pretreatment with bath application of MK-801 
A
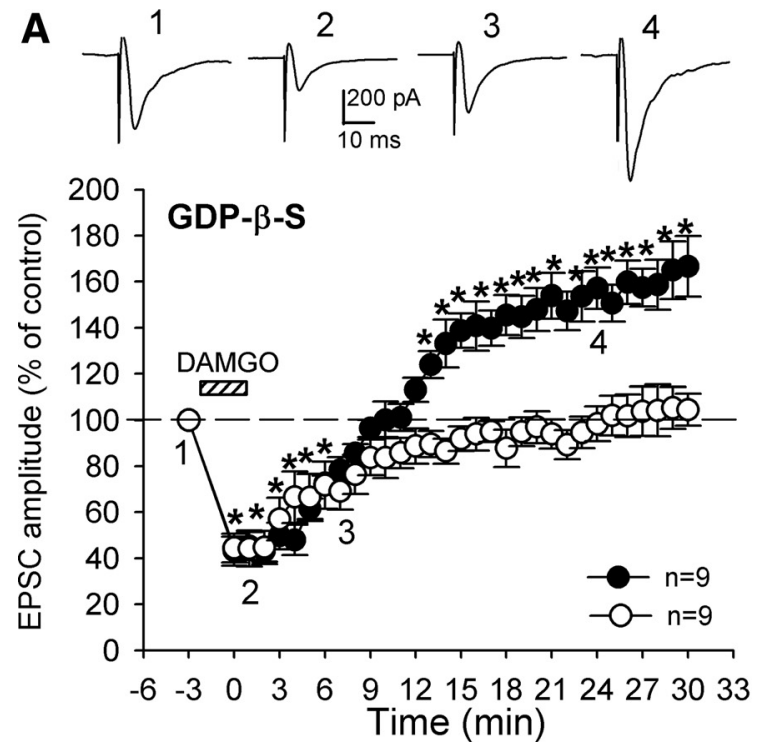

B

\section{Control (no GDP- $\beta-S)$}

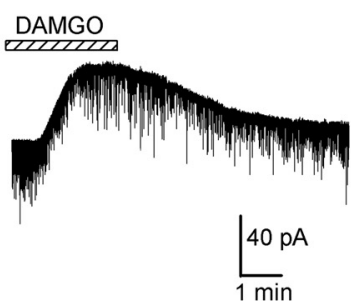

Intracellular GDP- $\beta-S$

DAMGO

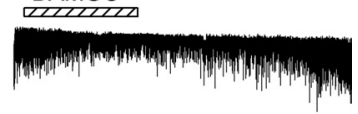

Figure 2. DAMGO induces LTP from primary afferent terminals in the spinal cord. A, Blocking the postsynaptic opioid action with GDP- $\beta$-S and $\mathrm{Cs}^{2+}$ failed to block LTP induction by DAMG0 in nine lamina II neurons without affecting the initial depression (black solid circles). In another nine neurons, DAMGO produced only acute depression (black open circles). Insets show individual EPSC traces of one neuron recorded at the time points indicated. ${ }^{*} p<0.05$ compared with the pre-DAMGO control value. $\boldsymbol{B}$, Representative traces show that blocking of postsynaptic opioid signaling with intracellular dialysis of $1 \mathrm{mM}$ GDP- $\beta$-S abolished the DAMG0-induced outward GIRK currents elicited by bath application of $1 \mu \mathrm{M}$ DAMGO in the lamina II neuron.
A
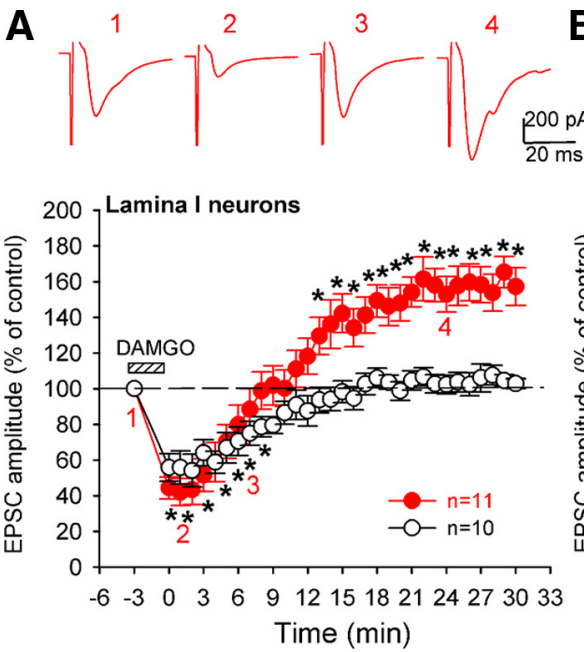
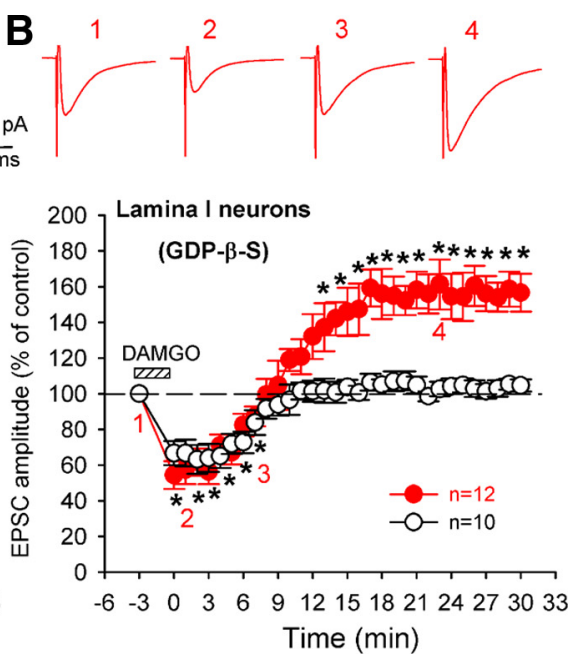

Figure 3. Opioid-induced LTP in lamina I neurons originates from primary afferent terminals in the spinal cord. $A$, Mean time courses of 11 lamina I neurons in which bath application of $1 \mu \mathrm{M}$ DAMGO for 3 min produced acute inhibition and LTP upon washout (red solid circles) and 10 neurons in which DAMG0 produced acute depression only (black open circles). B, Blocking the postsynaptic opioid signaling with GDP- $\beta$-S and $\mathrm{Cs}^{2+}$ failed to block the DAMGO-induced LTP in 12 lamina I neurons without affecting the initial depression (red solid circles). In another 10 neurons, DAMGO produced acute depression only (black open circles). Insets show individual EPSC traces of one neuron recorded at the time points indicated. Data are presented as mean \pm SEM. ${ }^{*} p<0.05$ compared with the pre-DAMGO control value.

(10 $\mu \mathrm{M})$ abolished DAMGO-induced LTP (data not shown). Additionally, bath application of $10 \mu \mathrm{M}$ MK-801 during the DAMGO washout period reversed DAMGO-induced LTP (Fig. 6D).

We also examined whether an increase in intracellular $\mathrm{Ca}^{2+}$, possibly resulting from NMDA receptor activation, plays a role in the opioid-induced LTP. In lamina II neurons, postsynaptic intracellular dialysis of the $\mathrm{Ca}^{2+}$ chelator BAPTA $(30 \mathrm{~mm})$ had no significant effect on the magnitude or incidence of DAMGO-induced LTP (Fig. 7A). However, bath application of the membrane-permeable $\mathrm{Ca}^{2+}$ chelator BAPTA-AM $(50 \mu \mathrm{M})$ abolished the DAMGO-induced LTP (Fig. 7B).

\section{Discussion}

The most salient findings of our study are that LTP induced after opioid cessation originates presynaptically in the spinal cord and that opioid exposure is capable of triggering sustained glutamate release from a population of primary afferent terminals. Our results suggest that both the acute depression and LTP produced by $\mu$-opioid receptor activation are presynaptic events. In both lamina I and II neurons, we found that $\sim 50 \%$ of the neurons displayed LTP upon DAMGO washout and that this phenomenon was abolished by the specific $\mu$-opioid receptor antagonist. Opioid-induced inhibition and LTP were associated with an increase in the paired-pulse depression ratio, which is a phenomenon commonly believed to reflect the synaptic release probability (Chen et al., 2004). We also found that, while intracellular dialysis of GDP- $\beta$-S completely blocked the postsynaptic GIRK currents elicited by $\mu$-opioid receptor activation, it had no effect on the opioidinduced LTP in either lamina I or lamina II neurons. Furthermore, blocking the postsynaptic NMDA receptors with MK801 or chelating $\mathrm{Ca}^{2+}$ in postsynaptic neurons with BAPTA failed to alter the opioid-induced LTP. However, bath application of NMDA receptor antagonists or BAPTA-AM abolished this LTP. Most importantly, we found that removing TRPV1-expressing primary sensory neurons not only abolished opioid-induced LTP but also produced a long-lasting inhibitory effect. Therefore, our findings provide strong evidence showing that opioid-induced LTP originates from a subpopulation of primary afferents that express TRPV1 and $\mu$-opioid receptors.

Ablation of TRPV1-expressing primary afferents not only abolished LTP but also induced a prolonged inhibition of EPSCs evoked from primary afferents and mEPSCs by DAMGO (i.e., LTD). Our findings could explain the prolonged opioid analgesic effect and resistance to the development of opioid tolerance found in RTX-treated rats (Chen and Pan, 2006; Chen et al., 2007). Because the initial inhibitory effect of DAMGO on evoked monosynaptic EPSCs was observed in all of the lamina I and II neurons tested, it suggests that C-fiber primary afferents expressing $\mu$-opioid receptors were stimulated in our study. Although measurement of mEPSCs alone cannot define the sources of presynaptic glutamate release, we found that RTX treatment abolished the DAMGO-induced increase in the mEPSC frequency. Thus, this finding suggests that increased synaptic glutamate release in 
A
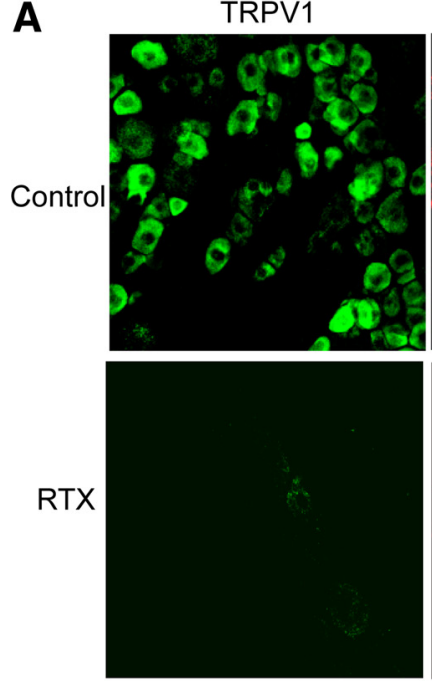

IB4
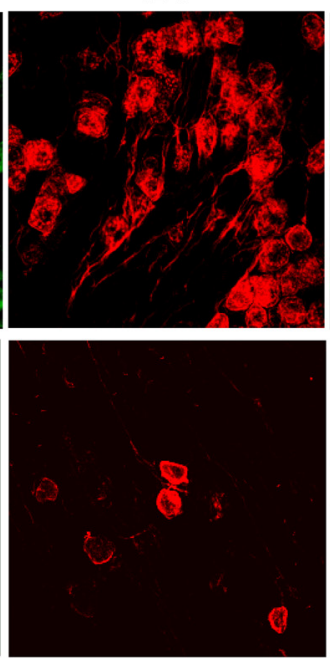
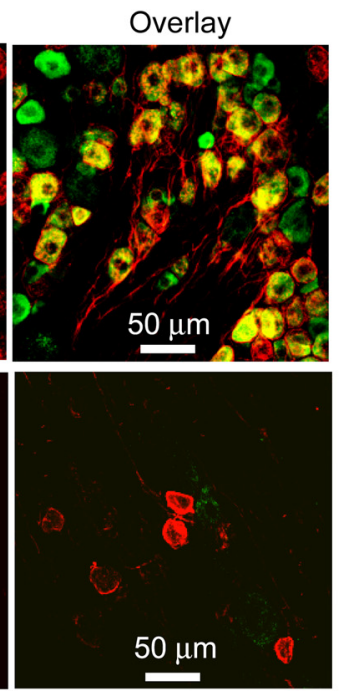

B
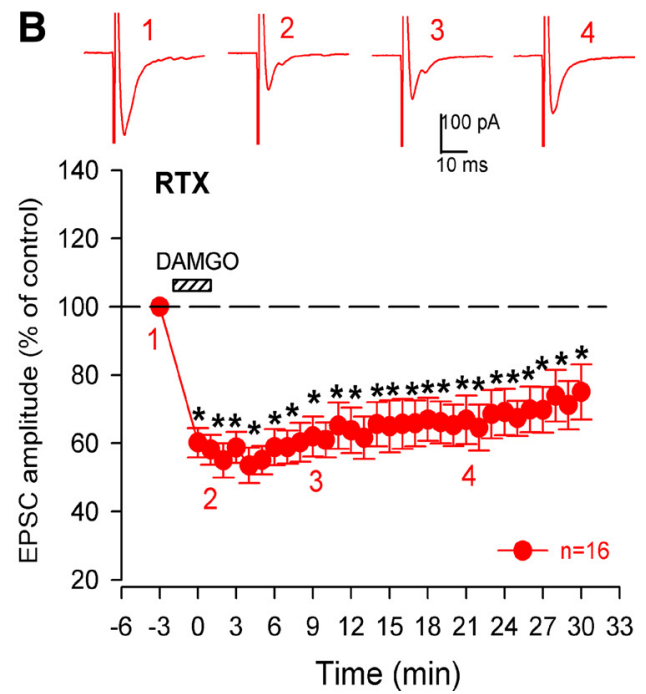

Figure 4. Opioid-induced presynaptic LTP originates from TRPV1-expressing primary afferents in the spinal cord. $A$, Treatment with the ultrapotent TRPV1 agonist RTX ablates dorsal root ganglion neurons. Representative confocal images from one RTX-treated and one vehicle control rat show TRPV1-immunoreactive and Griffonia simplicifolia isolectin $\mathrm{B}_{4}$ (IB $\mathrm{B}_{4}$, a marker of unmyelinated neurons)-positive neurons in the dorsal root ganglion. Colocalization of TRPV1 immunoreactivity and IB $\mathrm{B}_{4}$ labeling is indicated in yellow when the two images are digitally merged. All images are single confocal optical sections. $\boldsymbol{B}$, RTX treatment not only abolished LTP but also prolonged inhibition of the amplitude of evoked monosynaptic EPSCS by DAMGO in all of the 16 lamina II neurons tested. Insets show individuals traces of evoked EPSCS of one neuron recorded at the time points indicated. Data are presented as mean \pm SEM. ${ }^{*} p<0.05$ compared with the pre-DAMGO control value.

the spinal cord induced during opioid cessation originates from TRPV1-expressing primary afferent terminals. It remains unclear how removing TRPV1-expressing primary afferents leads to the LTD phenomenon after acute opioid exposure in the spinal cord. It is possible that primary afferent terminals with and without TRPV1 may interact directly or indirectly (via glutamatergic interneurons) in the superficial dorsal horn. Ablation of TRPV1-expressing primary afferents might have revealed the profound opioid inhibitory effect on non-TRPV1 primary afferents. In support of this view, we have shown that the $\mu$-opioid receptor agonists have a more profound inhibitory effect on voltage-gated $\mathrm{Ca}^{2+}$ channels in $\mathrm{IB}_{4}-$ negative than $\mathrm{IB}_{4}$-positive (most of them express TRPV1) dorsal root ganglion neurons (Wu et al., 2004). Because naloxone application during the DAMGO washout period does not readily reverse the longlasting inhibition of evoked EPSCs by DAMGO in RTX-treated rats (Zhou et al., 2008a), this LTD effect seems to be maintained by the intracellular signaling cascade after activation of $\mu$-opioid receptors located on non-TRPV1-expressing primary afferents.

In the spinal cord, NMDA receptors are present on primary afferent terminals (Liu et al., 1997), and the presynaptic NMDA receptors are involved in the increased glutamate release from primary afferents to lamina I neurons of young rats subjected to chronic opioid treatment (Zeng et al., 2006). LTP induction in the amygdala also depends on presynaptic, but not postsynaptic, NMDA receptors (Humeau et al., 2003). We found that pretreatment with bath application of NMDA receptor antagonists or
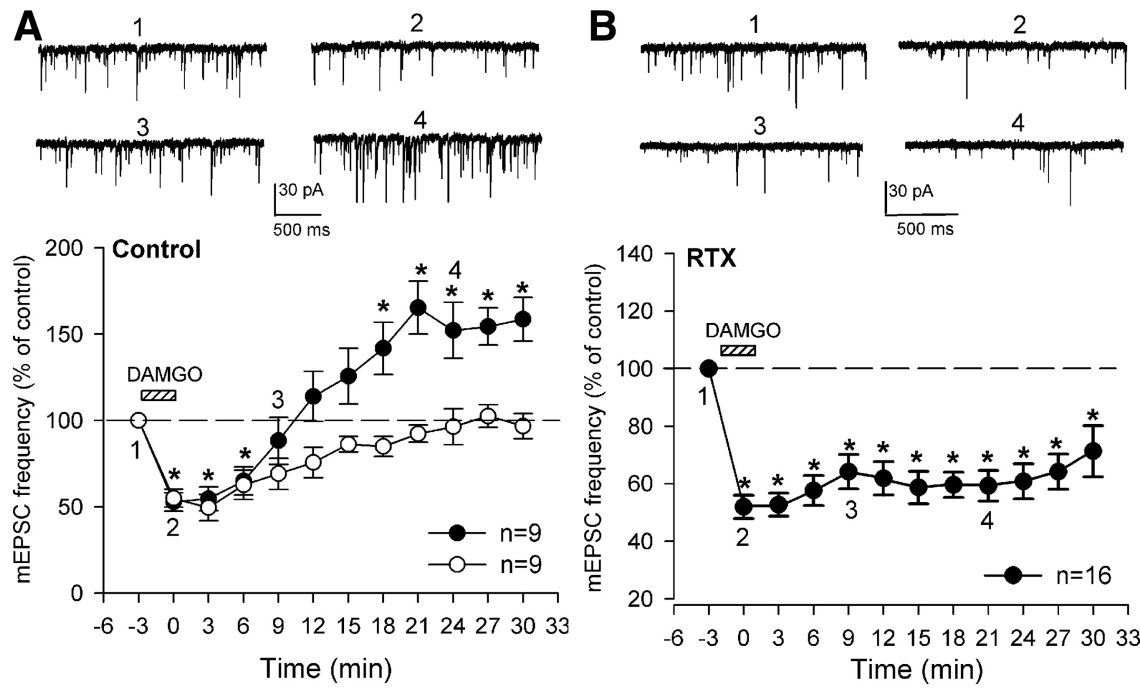

Figure 5. Opioid-induced synaptic glutamate release originates from TRPV1-expressing primary afferent terminals. A, Mean time courses of nine lamina II neurons where bath application of $1 \mu \mathrm{M}$ DAMGO for 3 min produced acute inhibition followed by potentiation of the frequency of mEPSCs upon washout (black solid circles) and another nine neurons in which DAMGO acutely inhibited mEPSCs only (black open circles). $\boldsymbol{B}$, RTX treatment not only abolished LTP but also prolonged inhibition of the frequency of $\mathrm{mEPSCS}$ by DAMGO in all of the 16 lamina II neurons tested. Insets show individuals traces of mEPSCs of one neuron recorded at the time points indicated. Data are presented as mean \pm SEM. ${ }^{*} p<0.05$ compared with the pre-DAMGO control value.

BAPTA-AM prevented the induction of LTP by DAMGO in the spinal cord. However, postsynaptic dialysis of MK-801 or BAPTA had no significant effects on either the magnitude or incidence of LTP. Furthermore, bath application of MK-801 during the DAMGO washout period reversed LTP, suggesting a pivotal role of NMDA receptors on TRPV1-expressing primary afferent terminals in the maintenance of opioid-induced LTP. Therefore, in addition to the presynaptic (i.e., inhibition of voltage-gated $\mathrm{Ca}^{2+}$ channels and neurotransmitter release) (Moises et al., 1994; Kohno et al., 1999) and postsynaptic (i.e., activation of GIRK) (Marker et al., 2005) effects involved in opioid analgesia, we propose that other presynaptic effects (i.e., stimulation of NMDA 

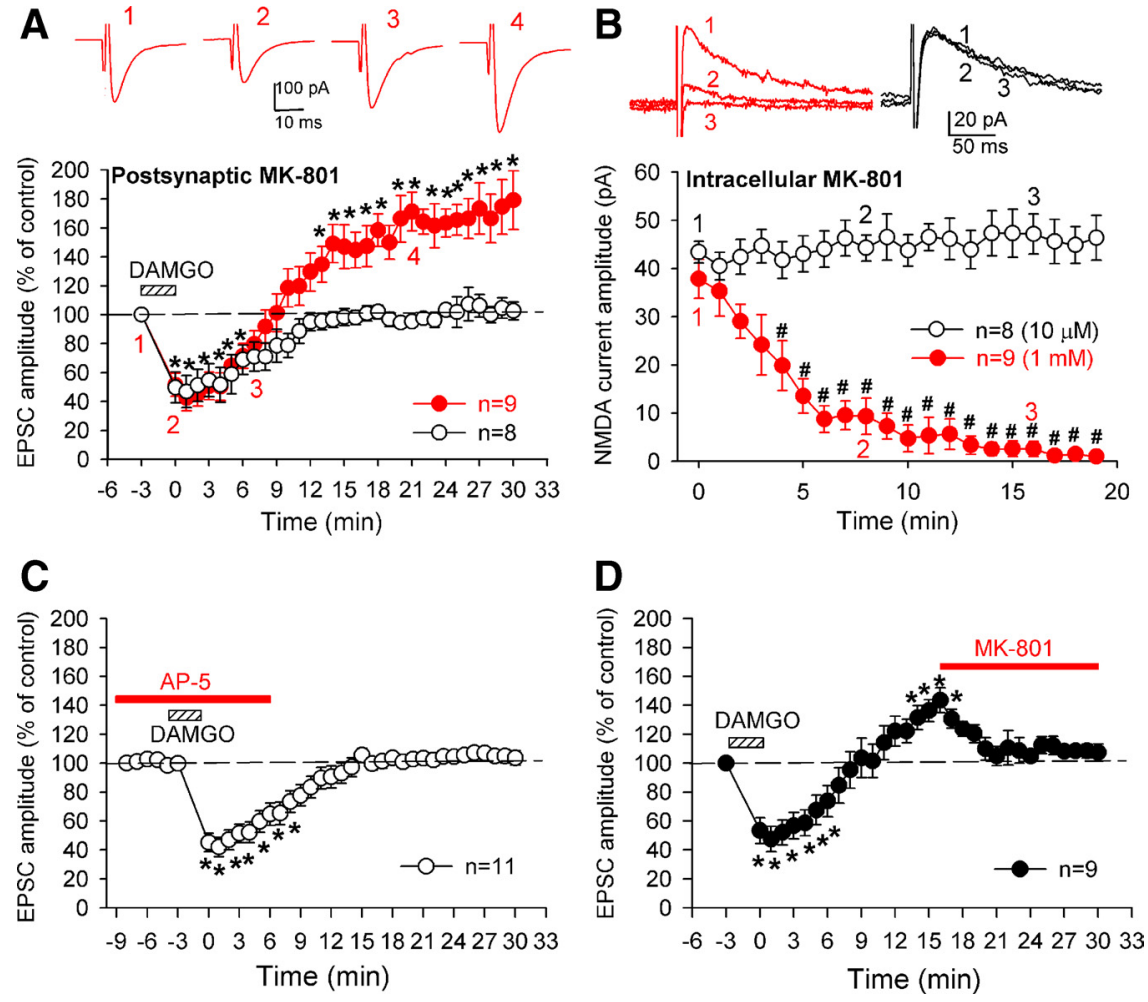

Figure 6. Presynaptic NMDA receptors contribute to opioid-induced LTP in the spinal cord. A, Postsynaptic dialysis with $1 \mathrm{~mm} \mathrm{MK-801}$ failed to block LTP induction by $1 \mu \mathrm{m}$ DAMGO in nine lamina II neurons (red solid circles). Insets show individual EPSC traces of one neuron recorded at the time points indicated. $\boldsymbol{B}$, Mean time courses of lamina II neurons where intracellular dialysis of $1 \mathrm{~mm}(n=9$ neurons, red solid circles), but not $10 \mu \mathrm{m}$ ( $n=8$ neurons, black open circles), of MK-801 blocked postsynaptic NMDA currents evoked from the dorsal root. Insets show individual NMDA current traces of neurons recorded at the time points indicated. C, Bath application of $50 \mu \mathrm{m} \mathrm{AP-5}$ before DAMG0 application blocked LTP induction without affecting the initial depression by DAMGO. D, Bath application of $10 \mu \mathrm{m} \mathrm{MK-801}$ during DAMG0-induced LTP reversed LTP in nine lamina II neurons. Data are presented as mean \pm SEM. ${ }^{*} p<0.05$ compared with the pre-DAMGO control value; ${ }^{\#} p<0.05$ compared with the control value at time 0 .
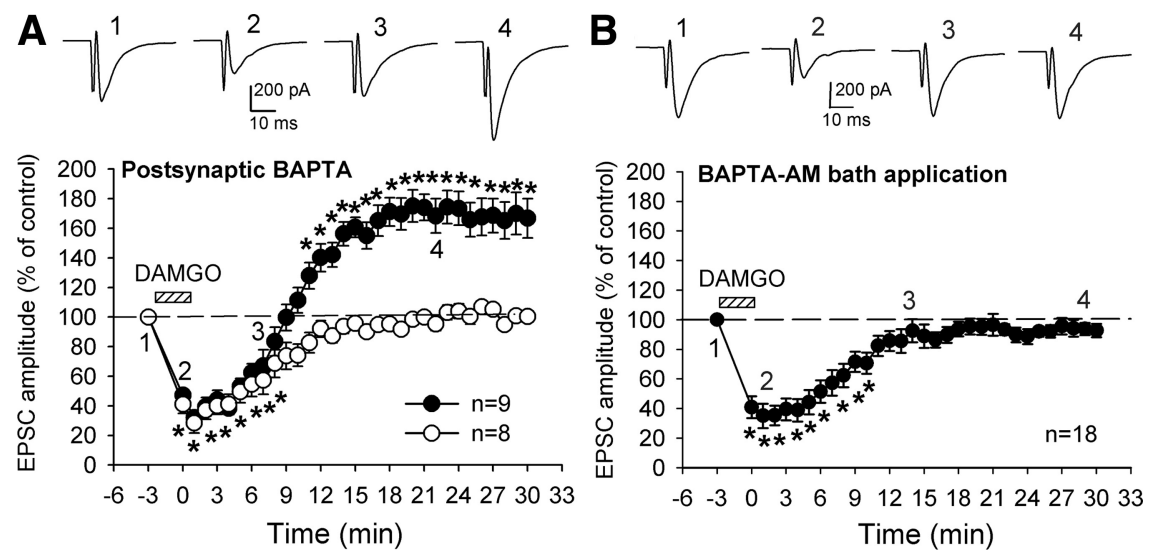

Figure 7. Increased intracellular calcium is involved in opioid-induced presynaptic LTP in the spinal cord. A, Postsynaptic dialysis with $30 \mathrm{~mm}$ BAPTA failed to block DAMGO (1 $\mu \mathrm{m}$ )-induced LTP in nine lamina II neurons (black solid circles). DAMGO produced acute inhibition only in another eight neurons dialyzed with BAPTA (black open circles). $\boldsymbol{B}$, Bath application of $50 \mu \mathrm{M}$ BAPTA-AM abolished DAMG0-induced LTP in 18 lamina II neurons without affecting the initial depression. Insets show individual monosynaptic EPSC traces of one neuron recorded at the time points indicated. Data are presented as mean \pm SEM. ${ }^{*} p<0.05$ compared with the pre-DAMGO control value.

receptors and increased $\mathrm{Ca}^{2+}$ levels) could increase the nociceptive input from TRPV1-expressing primary afferents and antagonize the analgesic effect of opioids. Further studies are warranted to reveal the intracellular signaling cascades that lead to increased neurotransmit- ter release from TRPV1-expressing primary afferents after acute and chronic opioid treatment.

Drdla et al. (2009) reported that LTP induced during the DAMGO washout period is mediated postsynaptically in the spinal cord. We noticed several problems with their study design. First, Drdla et al. only used five neurons for the GDP- $\beta$-S and MK-801 groups, and intracellular BAPTA application did not block opioidinduced LTP in all neurons in their study. Because lamina I and II neurons are highly heterogeneous and only $\sim 50 \%$ of these neurons displayed opioid-induced LTP, a larger sample size is required to minimize the possibility of sampling errors. Second, they used a recording condition (holding potential at $-70 \mathrm{mV}, 2 \mathrm{~mm} \mathrm{MgCl}_{2}$ in the pipette solution, and $1.3 \mathrm{mM} \mathrm{MgCl}_{2}$ in the bath solution) that is not optimal for studying postsynaptic NMDA receptors. The role of postsynaptic NMDA receptors in LTP induction is usually shown when the neuron is recorded at lower concentrations $(<0.5 \mathrm{~mm})$ of $\mathrm{Mg}^{2+}$ or by removing the $\mathrm{Mg}^{2+}$ block of the NMDA channel through direct depolarization of the cell or tetanic afferent stimulation (Bashir et al., 1991; Bender et al., 2006). However, Drdla et al. were able to record LTP using a recording condition that minimizes NMDA receptor activation, suggesting that postsynaptic NMDA receptors are not involved in opioid-induced LTP. Finally, $1000 \mu \mathrm{M}$ MK-801 in the pipette solution is required to block postsynaptic NMDA receptors (Humeau et al., 2003; Bender et al., 2006). Curiously, Drdla et al. showed that intracellular dialysis of $10 \mu \mathrm{M}$ MK-801 blocked LTP in all five neurons tested. However, as shown in our study, intracellular application of $10 \mu \mathrm{M} \mathrm{MK}$ 801 had no effect on postsynaptic NMDA currents in spinal dorsal horn neurons. Because MK-801 was used at such a low concentration that it cannot block postsynaptic NMDA receptors, the lack of LTP induction in this treatment group was probably caused by the sampling error in their study. Nevertheless, it should be noted that there are some differences between our study and theirs, such as the age of rats (7-8 weeks old versus $17-25$ d old) and concentration $(1 \mu \mathrm{M}$ versus $0.5 \mu \mathrm{M}$ ) and duration ( $3 \mathrm{~min}$ versus $15 \mathrm{~min}$ ) of DAMGO application. A number of postsynaptic signaling processes activated by opioids may take longer than 2-3 min to occur. For example, internalization/desensitization of opioid receptors and recovery of surface signaling does not occur if agonist exposure is too short (Walwyn et al., 2006; Arttamangkul et al., 2008; Virk and Williams, 2008). But it is less likely that the 
differences in the age and duration of DAMGO application can explain why a presynaptic LTP was not found in the study by Drdla et al. This is because in rats (12-17 d old) chronically treated with morphine for $3 \mathrm{~d}$, a presynaptic mechanism is responsible for increased glutamatergic input to spinal lamina I neurons (Zeng et al., 2006).

In summary, our study provides important new evidence that opioid-induced LTP in the spinal cord results from increased presynaptic glutamate release from TRPV1-expressing primary afferents. Increased dorsal horn neuronal activity after opioid exposure has been reported in vivo (Johnson and Duggan, 1981, 1984), but this rebound increase was precipitated by naloxone (not during the cessation of acute opioid treatment). Because animals do not exhibit any opioid withdrawal symptoms (typically precipitated by the opioid receptor antagonists) after acute opioid treatment, it is confusing to use the term opioid "withdrawal"-induced LTP. Based on the findings of our study, it seems that opioid-induced primary afferent hyperactivity is a more appropriate and precise term than LTP because it reflects both the functional state and cellular site in the spinal cord affected by the opioids. Thus, we propose that the term opioidinduced primary afferent hyperactivity should be used to describe increased glutamate release from primary afferents after acute opioid exposure. Chronic use of opioids can repeatedly stimulate TRPV1-expressing primary afferents and augment the nociceptive input, resulting in sensitization of spinal dorsal horn neurons and hyperalgesia. This sensitization can gradually overcome and mask the analgesic effects of the opioids. The critical role of TRPV1-expressing primary afferents in opioid-induced LTP has not been recognized previously, and this subpopulation of sensory neurons could be targeted to prevent or minimize opioid-induced hyperalgesia and tolerance.

\section{References}

Arttamangkul S, Quillinan N, Low MJ, von Zastrow M, Pintar J, Williams JT (2008) Differential activation and trafficking of micro-opioid receptors in brain slices. Mol Pharmacol 74:972-979.

Bashir ZI, Alford S, Davies SN, Randall AD, Collingridge GL (1991) Longterm potentiation of NMDA receptor-mediated synaptic transmission in the hippocampus. Nature 349:156-158.

Bender VA, Bender KJ, Brasier DJ, Feldman DE (2006) Two coincidence detectors for spike timing-dependent plasticity in somatosensory cortex. J Neurosci 26:4166-4177.

Célèrier E, Rivat C, Jun Y, Laulin JP, Larcher A, Reynier P, Simonnet G (2000) Long-lasting hyperalgesia induced by fentanyl in rats: preventive effect of ketamine. Anesthesiology 92:465-472.

Chen G, Harata NC, Tsien RW (2004) Paired-pulse depression of unitary quantal amplitude at single hippocampal synapses. Proc Natl Acad Sci U S A 101:1063-1068.

Chen SR, Pan HL (2006) Loss of TRPV1-expressing sensory neurons reduces spinal mu opioid receptors but paradoxically potentiates opioid analgesia. J Neurophysiol 95:3086-3096.

Chen SR, Prunean A, Pan HM, Welker KL, Pan HL (2007) Resistance to morphine analgesic tolerance in rats with deleted transient receptor potential vanilloid type 1-expressing sensory neurons. Neuroscience 145:676-685.

Chen Y, Geis C, Sommer C (2008) Activation of TRPV1 contributes to morphine tolerance: involvement of the mitogen-activated protein kinase signaling pathway. J Neurosci 28:5836-5845.

Devulder J (1997) Hyperalgesia induced by high-dose intrathecal sufentanil in neuropathic pain. J Neurosurg Anesthesiol 9:146-148.
Drdla R, Gassner M, Gingl E, Sandkühler J (2009) Induction of synaptic long-term potentiation after opioid withdrawal. Science 325:207-210.

Humeau Y, Shaban H, Bissière S, Lüthi A (2003) Presynaptic induction of heterosynaptic associative plasticity in the mammalian brain. Nature 426:841-845

Johnson SM, Duggan AW (1981) Evidence that the opiate receptors of the substantia gelatinosa contribute to the depression, by intravenous morphine, of the spinal transmission of impulses in unmyelinated primary afferents. Brain Res 207:223-228.

Johnson SM, Duggan AW (1984) Dependence in the absence of tolerance to morphine. Eur J Pharmacol 97:305-308.

Kohno T, Kumamoto E, Higashi H, Shimoji K, Yoshimura M (1999) Actions of opioids on excitatory and inhibitory transmission in substantia gelatinosa of adult rat spinal cord. J Physiol 518:803-813.

Li DP, Chen SR, Pan YZ, Levey AI, Pan HL (2002) Role of presynaptic muscarinic and $\mathrm{GABA}(\mathrm{B})$ receptors in spinal glutamate release and cholinergic analgesia in rats. J Physiol 543:807-818.

Liu H, Mantyh PW, Basbaum AI (1997) NMDA-receptor regulation of substance P release from primary afferent nociceptors. Nature 386:721-724.

Mao J, Price DD, Mayer DJ (1994) Thermal hyperalgesia in association with the development of morphine tolerance in rats: roles of excitatory amino acid receptors and protein kinase C. J Neurosci 14:2301-2312.

Marker CL, Luján R, Loh HH, Wickman K (2005) Spinal G-protein-gated potassium channels contribute in a dose-dependent manner to the analgesic effect of $\mu$ - and $\delta$ - but not $\kappa$-opioids. J Neurosci 25:3551-3559.

Moises HC, Rusin KI, Macdonald RL (1994) Mu- and kappa-opioid receptors selectively reduce the same transient components of high-threshold calcium current in rat dorsal root ganglion sensory neurons. J Neurosci 14:5903-5916.

Pan HL, Khan GM, Alloway KD, Chen SR (2003) Resiniferatoxin induces paradoxical changes in thermal and mechanical sensitivities in rats: mechanism of action. J Neurosci 23:2911-2919.

Pan YZ, Pan HL (2004) Primary afferent stimulation differentially potentiates excitatory and inhibitory inputs to spinal lamina II outer and inner neurons. J Neurophysiol 91:2413-2421.

Shen KF, Crain SM (1989) Dual opioid modulation of the action potential duration of mouse dorsal root ganglion neurons in culture. Brain Res 491:227-242.

Trujillo KA, Akil H (1991) Inhibition of morphine tolerance and dependence by the NMDA receptor antagonist MK-801. Science 251:85-87.

Van Elstraete AC, Sitbon P, Trabold F, Mazoit JX, Benhamou D (2005) A single dose of intrathecal morphine in rats induces long-lasting hyperalgesia: the protective effect of prior administration of ketamine. Anesth Analg 101:1750-1756.

Virk MS, Williams JT (2008) Agonist-specific regulation of mu-opioid receptor desensitization and recovery from desensitization. Mol Pharmacol 73:1301-1308.

Walwyn WM, Wei W, Xie CW, Chiu K, Kieffer BL, Evans CJ, Maidment NT (2006) $\mathrm{Mu}$ opioid receptor-effector coupling and trafficking in dorsal root ganglia neurons. Neuroscience 142:493-503.

Wu ZZ, Chen SR, Pan HL (2004) Differential sensitivity of N- and P/Q-type $\mathrm{Ca} 2+$ channel currents to a mu opioid in isolectin B4-positive and -negative dorsal root ganglion neurons. J Pharmacol Exp Ther 311:939-947.

Zeng J, Thomson LM, Aicher SA, Terman GW (2006) Primary afferent NMDA receptors increase dorsal horn excitation and mediate opiate tolerance in neonatal rats. J Neurosci 26:12033-12042.

Zhou HY, Chen SR, Chen H, Pan HL (2008a) Sustained inhibition of neurotransmitter release from non-TRPV1-expressing primary afferents by mu-opioid receptor activation-enkephalin in the spinal cord. J Pharmacol Exp Ther 327:375-382.

Zhou HY, Zhang HM, Chen SR, Pan HL (2008b) Increased C-fiber nociceptive input potentiates inhibitory glycinergic transmission in the spinal dorsal horn. J Pharmacol Exp Ther 324:1000-1010.

Zhou HY, Chen SR, Chen H, Pan HL (2009) The glutamatergic nature of TRPV1-expressing neurons in the spinal dorsal horn. J Neurochem 108: 305-318. 